\title{
Karnofsky performance score in acute renal failure as a predictor of short-term survival
}

Jose Ramon Perez Valdivieso, ${ }^{1}$ Maira Bes-Rastrollo, ${ }^{2}$ Pablo Monedero, ${ }^{1}$ Jokin De Irala ${ }^{2}$ and Francisco Javier Lavilla ${ }^{3}$

${ }^{1}$ Department of Anaesthesia and Critical Care, ${ }^{2}$ Department of Preventive Medicine and Public Health, and ${ }^{3}$ Renal Unit, University Hospital of Navarra, University of Navarra, Pamplona (Navarra), Spain

\section{SUMMARY}

Background: Karnofsky Performance Scale Index (KPS) is a measure of functional status that allows patients to be classified according to their functional impairment. We aim to assess if the prior KPS may predict the risk of death among patients with acute renal failure (ARF).

Methods: A cohort of 668 consecutive patients who had been admitted in an universityaffiliated hospital between June 2000 and June 2006, and had been diagnosed with ARF, were studied. Three hundred and eighty-six patients with ARF who matched at least one of the RIFLE (Risk, Injury, Failure, Loss and End stage) criteria on increased serum creatinine were included for subsequent analysis. The group was divided into four categories, according to different Karnofsky scores measured by a nephrologist $(\geq 80,70,60$ and $\leq 50)$. We used an adjusted logistic regression model to assess the relationship between the Karnofky score and mortality.

Results: A significant risk of in-hospital mortality within 90 days was observed when the other groups were compared with the $\geq 80$ Karnofsky group. Adjusted odds ratios were 8.87 (95\% confidence interval (CI) 3.03-25.99), 6.78 (95\% CI 2.61-17.58) and 2.83 (95\% CI 1.04-7.68), for Karnofsky groups of $\leq 50,60$ and 70, respectively. An adjusted odds ratio of 1.75 (95\% CI 1.37-2.23) was observed for every 10 point decrease in KPS score.

Conclusion: Functional status as indicated by the KPS is an independent predictor of death in this cohort of patients with ARF. Patients who presented lower scores had increased mortality rates.

Key words: acute renal failure, functional status, Karnofsky score, mortality, prognosis, RIFLE criteria.

Correspondence:

Dr Jose Ramon Perez Valdivieso

Department of Anaesthesia and Critical Care, Clinica Universitaria de Navarra, Pio XII, 36. 31008-Pamplona, Spain.

Email: jrpvaldi@unav.es 


\section{INTRODUCTION}

Despite advances in medical care, acute renal failure (ARF) remains a condition with a high mortality risk. ${ }^{1-9}$ Predicting the outcome can help to reassure patients and their families, and to address questions of efficiency and quality of care. ${ }^{10}$ It is therefore important to identify which patients may or not obtain benefits with more intensive treatments. Many prognostic scoring systems have been formulated to aid physicians to calculate outcomes in ARF. ${ }^{10-14}$ In addition to providing information about individual well-being at a given moment, the assessment of functional status could help identify those patients at risk for a worse outcome. ${ }^{15,16}$ It may be quickly and easily quantified by the Karnofsky Performance Scale Index (KPS) (Table 1), ${ }^{17,18}$ by studying the patient's activity, work and self-care. The KPS has been previously validated for oncological patients in Spain. ${ }^{19}$ The scale has been extensively used as an assessment tool in oncology since 1948, and it has been gaining interest as an independent risk factor for mortality in other clinical settings, as in patients with endstage renal disease ${ }^{20}$ or with glomerulonephritis due to systemic small vessel vasculitis. ${ }^{21}$ A modified version has been proposed as being useful in palliative care. ${ }^{22}$ The possible prognostic role of functional status for outcome in ARF has remained unproven, since it has been unknown whether coexisting morbidity and other prognostic variables might affect that association. The goal of our study was to assess if the Karnofsky score before admission to hospital could be considered an independent predictor of mortality in a cohort of patients with ARF treated by a nephrologist at a single centre.

\section{METHODS}

\section{Study population}

The University Hospital of Navarra is a tertiary care teaching medical centre with 400 beds in the city of Pamplona, Spain. Hospital ethics committee approval was obtained. A cohort of 668 patients with ARF, who had been admitted at our hospital between 2000 and 2006, was prospectively studied. We restricted our analysis to patients with adequate increment of creatinine to qualify for the RIFLE (Risk, Injury, Failure, Loss and End stage) criteria for ARF, ${ }^{23}$ and who were older than 16 years old.

\section{Definition of the variables}

Functional status before admission was measured through the KPS by one nephrologist. Eleven qualifying items were measured, ranging from a score of 0 for death to 100 for normal function, higher scores signified better functional status. Recently, the Acute Dialysis Quality Initiative (ADQI) workgroup proposed a consensus definition of ARF, using a set of criteria called the RIFLE criteria. ${ }^{23}$ The RIFLE criteria provide a graded definition of ARF severity (Risk, Injury and Failure), stratifying patients according to relative changes of serum creatinine and urine output. Patients were classified to the maximum RIFLE class (Risk, Injury or Failure) according to their creatinine levels only. We were unable to collect full information for urine output. The peak creatinine was defined as the highest creatinine during their admission. For patients without chronic renal failure reported, the baseline creatinine was calculated using the Modification of Diet in Renal Disease (MDRD) equation, ${ }^{24}$ as recommended by the ADQI, ${ }^{23}$ assuming a glomerular filtration rate of $75 \mathrm{~mL} / \mathrm{min}$ per $1.73 \mathrm{~m}^{2}$. For patients with a history of renal failure, the baseline creatinine was defined as the one measured 
at hospital admission. Demographic data, aetiology of ARF, currently comorbid conditions and laboratory data were all prospectively collected. Comorbid conditions were used to assess the severity of illness, that was measured through Liano score $(0.032 \mathrm{x}$ age in decades $-0.086 \mathrm{x}$ male gender $-0.109 \mathrm{x}$ nephrotoxic $+0.109 \mathrm{x}$ oliguria $+0.116 \times$ hypotension $+0.122 \times$ jaundice $+0.150 \times$ coma $-0.154 \times$ consciousness + $0.182 \mathrm{x}$ assisted respiration +0.210$)$. ${ }^{10}$ The difference between serum creatinine value obtained at the time of the nephrology consultation and the baseline value was calculated and expressed in percentage (\%): Relative creatinine change $(\%)=$ [(creatinine when nephrologist saw the case - basal creatinine)/basal creatinine] x 100 . Food intake was defined as the caloric ingestion in the previous days, and this was classified as 'appropriate' when it was optimal, 'mild malnutrition' when it had been inadequate for less than 3 days, 'moderate malnutrition' when it had been inadequate between 3 and 7 days, and 'severe malnutrition' when it had been inadequate for more than 7 days. The patients' clinical status and treatment for ARF were recorded daily. We continued follow up to study the mortality at 90 days from the beginning of nephrology consultation.

Serum creatinine concentration was quantified using the kinetic Jaffe assay.

All cases were treated during the admission at our hospital by the same nephrologist and all the data were gathered by the same observer.

The primary outcome variable was in-hospital mortality within 90 days.

\section{Statistical analysis}

Our population was divided into four categories regarding four Karnofsky scores that might be clinically significant and to facilitate the statistical analysis $(\geq 80,70,60$ and $\leq 50)$. The reason for selecting four Karnofsky categories was mainly because they discriminate between four performance statuses that might be useful in the clinical practice. The score $\geq 80$ shows patients who are able to carry on normal activity; the score 70 shows patients who, despite the inability for normal activity, can take care of themselves; the score 60 shows an important difference in means of self-care, patients need some kind of assistance, though occasional; while the score $\leq 50$ has particular importance because it shows patients who need frequent medical care and assistance.

We considered the Karnofsky scoring before the hospital admission as the exposure variable. Non-conditional logistic regression models were fitted to assess the relationship between the Karnofsky scoring and the risk of in-hospital mortality. Odds ratios (OR) and their 95\% confidence intervals (CI) were calculated considering the group of Karnofsky scores of $\geq 80$ as the reference category.

Continuous variables were expressed as medians (and interquartile ranges), and compared using ANOVA test. We fitted a crude model (univariate, i.e. without any adjustment), an age and gender model, and a multivariate-adjusted model including the following variables: Liano scoring, percentage of the difference between serum creatinines, prior food intake, whether patients were oncological or not, treatment of ARF and RIFLE classes selected by the descriptive analysis of potential confounders. The Liano scoring includes in its equation the variables for nephrotoxicity, oliguria, hypotension, jaundice, mental status and assisted respiration. Categorical variables were expressed as proportions and compared with the chi-squared test. We evaluated all firstorder multiplicative interactions (effect modifications) through product terms. The area 
under the receiver operating characteristic (ROC) curve was calculated to assess the performance of the model. ${ }^{25}$

We analysed overall survival at 90 days across groups using the Kaplan-Meier methods, and tested differences between groups using the log-rank test. The time of origin was the date when the nephrology consultation started. The event defined was death, whereas those cases alive at the end of follow up and those lost to follow up were censored at their last observation.

All P-values presented are two-tailed, $\mathrm{P}<0.05$ was considered statistically significant. Statistical analyses were conducted using SPSS version 10.0.7 (SPSS Inc., Chicago, IL, USA).

\section{RESULTS}

We evaluated 668 patients. Of these, 219 were excluded because they failed to fulfil the RIFLE criteria for serum creatinine values and were not oliguric; 28 presented oliguria (but it could not be measured on a $6 \mathrm{~h}$ basis) but did not fulfil the creatinine criteria; five patients were under 16 years old; and 30 patients had incomplete records. So, 386 patients remained. They were divided into four groups according to Karnofsky scores $(\geq 80,70,60$ and $\geq 50)$. Demographics and baseline clinical characteristics are shown in Table 2. The groups were similar in proportions of diabetic, surgical and known chronic renal failure patients. They were also similar in terms of age, gender, causes of ARF and basal serum albumin. The lower Karnofsky score presented a higher mortality rate. There were some significant differences between the groups of Karnofsky scores that could be relevant in the clinical setting: The lower groups showed higher previous increment of serum creatinine $(\mathrm{P}=0.013)$, worse Liano scores and some elements from its equation, lower basal haemoglobin, more impaired prior to food intake, lesser proportion of oncology patients, and were given more invasive treatment. The Liano scoring includes in its equation the variables for nephrotoxicity, oliguria, hypotension, jaundice, mental status, and assisted respiration. Higher mortality rates were presented in those groups with higher increments of creatinine, worst Liano scoring, lesser levels of haemoglobin, impaired prior food intake or more aggressive treatment. Adjustment for covariates was performed. Results of the multiple-variable logistic regression analyses examining the impact of the different Karnofsky scores for in-hospital mortality is shown in Table 3. We adjusted for potential confounders (age, sex, percentage of difference between serum creatinines, Liano scoring, RIFLE classes, food intake, basal haemoglobin, oncological patients and treatment). Patients with scores of $\leq 50,60$ and 70 presented adjusted ORs of 8.87 (95\% CI 3.03-25.99), 6.78 (95\% CI 2.61-17.58) and 2.83 (95\% CI 1.04-7.68), respectively, compared with those with scores of $\geq 80$. The OR for mortality associated with every 10 points of KPS decrease was 1.75 (95\% CI 1.37-2.23) (Table 4).

ROC curves analyses were calculated (Fig. 1). The area under the curve was 0.701 .

We analysed the short-term survival. According to the Kaplan-Meier plot, the lower Karnofsky scores also presented a statistically significant higher mortality rate in the period between the nephrology consultation and 90 days (Fig. 2) compared with Karnofsky scores of $\geq 80(\log -$ rank test $=0.001)(30,52,17$ and 7 deaths for Karnofsky groups of $\leq 50,60,70$ and $\geq 80$, respectively). Most of the deaths (28, 38, 17 and 3 
deaths for Karnofsky groups of $\leq 50,60,70$ and $\geq 80$, respectively) occurred during the first 60 days of follow up.

\section{DISCUSSION}

This study involved a cohort of non-oncological and oncological patients and sought to examine the relationship between the prior functional status with the risk of mortality in ARF. The KPS has been regarded as the gold standard measurement of performance status in cancer, but it has demonstrated also to be useful in other clinical settings. ${ }^{20-22}$ We found that Karnofsky scoring had an independent influence on mortality in this cohort. Comparing Karnofsky scores, patients with counts of $\leq 50,60$ and 70 had rates of in-hospital mortality of $48.4 \%, 39.7 \%$ and $21.6 \%$, respectively, versus rates of $7.7 \%$ among patients with the highest Karnofsky scores $(\geq 80)$. We believe it is important to note that a relative increment risk for mortality of $75 \%$ was observed for every 10 points of KPS decrease, after adjusting for other comorbility factors. Although we cannot conclude what the causes of the poorer functional status were presenting all the patients' similar ages, we suspect that common pathological conditions as obesity, chronic obstructive pulmonary disease, chronic pain or cardiovascular diseases might have been involved.

The association between functional status and mortality might reinforce the common belief that frail patients do poorly when they suffer a serious illness. Measurement of KPS presented at home before hospital admission may be useful. It could improve the prediction of the risk of death among patients with ARF.

Because of heterogeneous nature of ARF, it was important to adjust for the severity of illness $^{26}$ with Liano scoring, and for the acute kidney injury using the RIFLE criteria. We have chosen the Liano score because of its ability to discriminate mortality from survival and its ability to calibrate the observed mortality rate with the expected mortality in ARF.,26,27 Adjusting the severity of illness and using the RIFLE criteria will allow more meaningful comparisons in future studies on ARF, ${ }^{6,23,26}$ and is one of the main strengths of this study. Patients did not do worse because they had more severe ARF. Those patients with the same RIFLE class and Liano scores, but higher KPS, presented lower mortality rates.

It is of interest to point out that 217 patients from the original population would not have been diagnosed with ARF according to the RIFLE criteria. They did not present oliguria or enough creatinine increment, but were diagnosed of ARF according to other criteria at that time.

The ROC curve showed an area of 0.701 . The conventional threshold of 0.7 has been typically considered satisfactory for clinical use. ${ }^{28}$

There are several limitations to this study. As an observational single centre study, the results identified and described the association between KPS and mortality only. These results should be validated with further investigations. With larger numbers and from several centres the CIs would decrease in range. Nevertheless, the trend towards increased mortality is strong. We believe that this study may prompt larger studies. Selection bias may have influenced the results; however, objective data were collected 
by a single investigator following strict criteria, and reviewing the data. Urine output data were not available; therefore, it was not possible to estimate the RIFLE criteria according to this value. We started to collect the data before the RIFLE criteria was formulated, and most of the ward patients had no urine output measured on a six-hourly basis. We assume that we might underestimate some cases according to the RIFLE criteria. When those excluded oliguric patients $(n=28)$ were included to the analysis into another RIFLE category, an increase of our estimates occurred in the multivariate model (OR 10.52, 95\% CI 3.78-29.27; OR 8.35, 95\% CI 3.24-21.53; OR 3.09, 95\% CI 1.13-8.41, for Karnofsky groups of $\leq 50,60$ and 70 , respectively). Thus, misclassification of this covariate would most likely bias the ORs towards the null value. Although we cannot rule out the possibility of residual confounding, it is not likely that the strong association found could be explained by such a residual confounding. Moreover, we adjusted for potential risk factors for mortality among ARF patients.

We should concede that a limited but indetermined number of patients might have been readmitted after hospital discharge. In that situation, they were included in the cohort as new patients. Their KPS and other variables were measured again.

We calculated the MDRD equation to obtain a baseline serum creatinine in patients with no previous history of renal failure because a true baseline is often unknown. We solved the equation assuming a GFR of $75 \mathrm{~mL} / \mathrm{min}$ per $1.73 \mathrm{~m}^{2}$, which has been reported to estimate a lower limit of a normal GRF., ${ }^{4,6,23}$ The MDRD formula may be less accurate at normal GFR, resulting in overdiagnosis of ARF; however, it is a commonly used test in clinical settings where ARF is a concern. The MDRD also has the advantage of not requiring body weight data. ${ }^{29} \mathrm{We}$ analysed mortality within 90 days. It could be argued that all-cause mortality at longer time is unrelated to. Considering 90 days of follow up, the KPS remained significantly associated with mortality. These findings agree with previous reports and recommendations about optimal follow-up time in patients with established ARF. ${ }^{23,30,31}$ Unfortunately, discharged patients were not followed up within that time period, so we can only present in-hospital mortality rates.

This study does not provide any direct improvement in the treatment of ARF. However, it gives more information about the utility of measuring KPS as an inexpensive, noninvasive and independent prognosis tool to identify highrisk ARF patients. It also strengthens the importance of improving the functional status in the oncology and general population. The unchanged mortality rates of ARF over the past years might have been affected by the constant increment of frailer patients, despite technical advances.

\section{CONCLUSION}

In this cohort of patients, the lower scores for the KPS were strongly associated with higher risk of death, independent of ARF severity, comorbid illnesses and other possible confounders. The use of this simple and rapid scale at the start of the treatment could aid clinicians to improve the knowledge of the risk profile of every patient with ARF. Our findings highlight the importance of functional status on clinical outcomes in this disease. Assessment of functional status should be taken into account to compare patients in future clinical studies, and adjust for it in the analysis when necessary. 


\section{ACKNOWLEDGEMENTS}

The authors wish to express their gratitude for the contributions made by Dr Leonard Yuen. This study was part of our daily clinical work.

\section{REFERENCES}

1. Abosaif NY, Tolba YA, Heap M, Russell J, El Nahas AM. The outcome of acute renal failure in the intensive care unit according to RIFLE: Model application, sensitivity, and predicability. Am. J. Kidney Dis. 2005; 46: 1038-48.

2. Hegarty J, Middleton RJ, Krebs $M$ et al. Severe acute renal failure in adults: Place of care, incidence and outcomes. QJM 2005; 98: 661-6.

3. Herrera-Gutierrez ME, Seller-Perez G, Maynar-Moliner J, Sanchez-IzquierdoRiera JA. [Epidemiology of acute kidney failure in Spanish ICU. Multicenter prospective study FRAMI]. Med. Intensiva 2006; 30: 260-67.

4. Hoste EA, Clermont G, Kersten A et al. RIFLE criteria for acute kidney injury are associated with hospital mortality in critically ill patients: A cohort analysis. Crit. Care 2006; 10: R73.

5. Uchino S. The epidemiology of acute renal failure in the world. Curr. Opin. Crit. Care 2006; 12: 538-43.

6. Uchino S, Bellomo R, Goldsmith D, Bates S, Ronco C. An assessment of the RIFLE criteria for acute renal failure in hospitalized patients. Crit. Care Med. 2006; 34: 1913-17.

7. Uchino $\mathrm{S}$, Bellomo R, Morimatsu $\mathrm{H}$ et al. External validation of severity scoring systems for acute renal failure using a multinational database. Crit. Care Med. 2005; 33: 1961-7.

8. Uchino S, Kellum JA, Bellomo $\mathrm{R}$ et al. Acute renal failure in critically ill patients: A multinational, multicenter study. JAMA 2005; 294: 813-18.

9. Ympa YP, Sakr Y, Reinhart K, Vincent JL. Has mortality from acute renal failure decreased? A systematic review of the literature. Am. J. Med. 2005; 118 : 827-32.

10. Liano F, Gallego A, Pascual $J$ et al. Prognosis of acute tubular necrosis: An extended prospectively contrasted study. Nephron 1993; 63: 21-31.

11. Chertow GM, Lazarus JM, Paganini EP, Allgren RL, Lafayette RA, Sayegh MH. Predictors of mortality and the provision of dialysis in patients with acute tubular necrosis. The Auriculin Anaritide Acute Renal Failure Study Group. J. Am. Soc. Nephrol. 1998; 9: 692-8.

12. Dharan KS, John GT, Antonisamy B, Kirubakaran MG, Jacob CK. Prediction of mortality in acute renal failure in the tropics. Ren. Fail. 2005; 27: 289-96.

13. Mehta RL, Pascual MT, Gruta CG, Zhuang S, Chertow GM. Refining predictive models in critically ill patients with acute renal failure. J. Am. Soc. Nephrol. 2002; 13: 1350-57.

14. Paganini EP, Halstenberg WK, Goormastic M. Risk modeling in acute renal failure requiring dialysis: The introduction of a new model. Clin. Nephrol. 1996; 46: 206-11.

15. Valdes C, Garcia-Mendoza M, Rebollo P, Ortega T, Ortega F. Mental health at the third month of haemodialysis as a predictor of short-term survival. Nephrol. Dial. Transplant. 2006; 21: 3223-30. 
16. Lopez Revuelta K, Garcia Lopez FJ, de Alvaro Moreno F, Alonso J. Perceived mental health at the start of dialysis as a predictor of morbidity and mortality in patients with end-stage renal disease (CALVIDIA Study). Nephrol. Dial. Transplant. 2004; 19: 2347-53.

17. Karnofsky DA, Burchenal J (eds.). The Clinical Evaluation of Chemotherapeutic Agents in Cancer. New York: Columbia University Press, 1949.

18. Mor V, Laliberte L, Morris JN, Wiemann M. The Karnofsky Performance Status Scale. An examination of its reliability and validity in a research setting. Cancer 1984; 53: 2002-7.

19. Agra Varela Y, Badia Llach X, Gil Miguel A. [Instrument for the measure of quality of life in cancer patients]. Med. Clin. 1998; 110: 703-8.

20. McClellan WM, Anson C, Birkeli K, Tuttle E. Functional status and quality of life: Predictors of early mortality among patients entering treatment for end stage renal disease. J. Clin. Epidemiol. 1991; 44: 83-9.

21. Little MA, Nazar L, Farrington K. Outcome in glomerulonephritis due to systemic small vessel vasculitis: Effect of functional status and non-vasculitic co-morbidity. Nephrol. Dial. Transplant. 2004; 19: 356-64.

22. Abernethy AP, Shelby-James T, Fazekas BS, Woods D, Currow DC. The Australia-modified Karnofsky Performance Status (AKPS) scale: A revised scale for contemporary palliative care clinical practice [ISRCTN81117481]. BMC Palliat. Care 2005; 4: 7.

23. Bellomo R, Ronco C, Kellum JA, Mehta RL, Palevsky P. Acute renal failure definition, outcome measures, animal models, fluid therapy and information technology needs: The Second International Consensus Conference of the Acute Dialysis Quality Initiative (ADQI) Group. Crit. Care 2004; 8: R204-12.

24. National Kidney Foundation. K/DOQI clinical practice guidelines for chronic kidney disease: Evaluation, classification, and stratification. Am. J. Kidney Dis. 2002; 39 (2 Suppl. 1): S1-S266.

25. Hosmer D, Lemeshow S. Applied Logistic Regression. New York: Wiley, 1989.

26. Star RA. Treatment of acute renal failure. Kidney Int. 1998; 54: 1817-31.

27. Douma CE, Redekop WK, van der Meulen JH et al. Predicting mortality in intensive care patients with acute renal failure treated with dialysis. J. Am. Soc. Nephrol. 1997; 8: 111-17.

28. Lemeshow S, Le Gall JR. Modeling the severity of illness of ICU patients. A systems update. JAMA 1994; 272: 1049-55.

29. Lameire N, Adam A, Becker CR et al. Baseline renal function screening. Am. J. Cardiol. 2006; 98 (6A): 21K-6K.

30. Bell M, Liljestam E, Granath F, Fryckstedt J, Ekbom A, Martling CR. Optimal follow-up time after continuous renal replacement therapy in actual renal failure patients stratified with the RIFLE criteria. Nephrol. Dial. Transplant. 2005; 20: 354-60.

31. Palevsky PM, Metnitz PG, Piccinni P, Vinsonneau C. Selection of endpoints for clinical trials of acute renal failure in critically ill patients. Curr. Opin. Crit. Care 2002; 8: 515-18. 


\begin{tabular}{|l|l|}
\hline \multicolumn{2}{|l|}{ Table 1. Karnofsky Performance Scale Index (KPS) } \\
\hline Score (category) & Karnofsky \\
\hline 100 & $\begin{array}{l}\text { Normal; no complaints; no evidence of } \\
\text { disease. }\end{array}$ \\
\hline 90 & $\begin{array}{l}\text { Able to carry on normal activity; minor } \\
\text { signs or symptoms. }\end{array}$ \\
\hline 80 & $\begin{array}{l}\text { Normal activity with effort; some signs or } \\
\text { symptoms of disease. }\end{array}$ \\
\hline 70 & $\begin{array}{l}\text { Care for self; unable to carry on normal } \\
\text { activity or to do active work. }\end{array}$ \\
\hline 60 & $\begin{array}{l}\text { Requires occasional assistance but is able } \\
\text { to care for most of his needs. }\end{array}$ \\
\hline 50 & $\begin{array}{l}\text { Requires considerable assistance and } \\
\text { frequent medical care. }\end{array}$ \\
\hline 40 & $\begin{array}{l}\text { Disabled; requires special care and } \\
\text { assistance. }\end{array}$ \\
\hline 30 & $\begin{array}{l}\text { Severely disabled; hospitalization } \\
\text { necessary; active supportive treatment is } \\
\text { necessary. }\end{array}$ \\
\hline 20 & $\begin{array}{l}\text { Very sick; hospitalization necessary; } \\
\text { active supportive treatment is necessary. }\end{array}$ \\
\hline 10 & $\begin{array}{l}\text { Moribund; fatal processes progressing } \\
\text { rapidly. }\end{array}$ \\
\hline Dead. \\
\hline
\end{tabular}


Table 2. Demographics and baseline clinical characteristics of patients

\begin{tabular}{|c|c|c|c|c|c|}
\hline Variables* & $\begin{array}{c}\text { Karnofsky } \leq 50 \\
(n=62)\end{array}$ & $\begin{array}{c}\text { Karnofsky }=60 \\
(n=136)\end{array}$ & $\begin{array}{c}\text { Karnofsky }=70 \\
(n=97)\end{array}$ & $\begin{array}{c}\text { Karnofsky } \geq 80 \\
(n=91)\end{array}$ & $P$-value \\
\hline Oncology patients $(\%)$ & 32.3 & 44.9 & 39.2 & 54.9 & 0.021 \\
\hline Mortality $(\%)$ & 48.4 & 39.7 & 21.6 & 7.7 & $<0.001$ \\
\hline $\begin{array}{l}\text { Increase of Creatinine\#: median \% } \\
\text { (IQR) }\end{array}$ & $92.99(134.83)$ & $122.99(174.43)$ & $97.68(207.31)$ & $66.20(142.86)$ & 0.013 \\
\hline Age: median years (IQR) & $60.50(19.75)$ & $65.00(17.00)$ & $63.00(19.00)$ & $59.00(22.00)$ & 0.055 \\
\hline Male gender $(\%)$ & 71.0 & 69.1 & 64.9 & 73.6 & 0.80 \\
\hline Surgical (\%) & 25.6 & 19.8 & 30.8 & 29.9 & 0.20 \\
\hline RIFLE criteria (\%) & & & & & 0.05 \\
\hline Risk & 24.2 & 14.7 & 18.6 & 33.0 & \\
\hline Injury & 30.6 & 27.9 & 25.8 & 29.7 & \\
\hline Failure & 45.2 & 57.4 & 55.7 & 37.4 & \\
\hline Liano scoring: median score (IQR) & $0.40(0.36)$ & $0.30(0.33)$ & $0.25(0.30)$ & $0.19(0.20)$ & $<0.001$ \\
\hline $\begin{array}{l}\text { Basal serum albumin (mg/dL): median } \\
(\mathrm{IQR})\end{array}$ & $2.4(1.1)$ & $2.4(1.0)$ & $2.6(1.0)$ & $2.6(0.8)$ & 0.13 \\
\hline $\begin{array}{l}\text { Basal haemoglobin (g/dL): median } \\
\text { (IQR) }\end{array}$ & $10.15(3.35)$ & $10.50(3.10)$ & $10.70(3.48)$ & $11.40(3.13)$ & 0.036 \\
\hline $\begin{array}{l}\text { Patients with history of chronic renal } \\
\text { failure }(\%)\end{array}$ & 21.0 & 26.5 & 24.7 & 20.9 & 0.75 \\
\hline Diabetic $(\%)$ & 6.5 & 8.8 & 6.2 & 8.8 & 0.83 \\
\hline Aminoglycoside use (\%) & 21 & 16.9 & 6.2 & 9.9 & 0.009 \\
\hline Radiocontrast procedure (\%) & 1.6 & 16.2 & 8.2 & 22.0 & 0.007 \\
\hline Hypotension (\%) & 53.2 & 39.7 & 37.1 & 25.3 & 0.001 \\
\hline Oliguria (\%) & 43.5 & 51.5 & 40.2 & 19.8 & $<0.001$ \\
\hline Jaundice $(\%)$ & 50.0 & 32.4 & 24.7 & 18.7 & $<0.001$ \\
\hline Coma $(\%)$ & 17.7 & 9.6 & 7.2 & 4.4 & 0.006 \\
\hline Consciousness $(\%)$ & 77.4 & 83.8 & 86.6 & 86.8 & 0.123 \\
\hline Mechanical ventilation (\%) & 29.0 & 19.1 & 19.6 & 15.4 & 0.075 \\
\hline Nephrotoxicity (\%) & 40.3 & 36.8 & 36.1 & 56.0 & 0.030 \\
\hline Causes of acute renal failure (\%) & & & & & 0.38 \\
\hline Pre-renal & 91.9 & 77.9 & 81.4 & 76.9 & \\
\hline Intrinsic renal & 8.1 & 14.7 & 13.4 & 20.9 & \\
\hline Post-renal & 0.0 & 2.2 & 4.1 & 1.1 & \\
\hline Other causes & 0.0 & 5.1 & 1.0 & 1.1 & \\
\hline Treatment of acute renal failure (\%) & & & & & $<0.001$ \\
\hline Non-dialytic & 64.5 & 68.4 & 70.1 & 84.6 & \\
\hline Intermittent haemodialysis & 0.0 & 5.9 & 5.2 & 5.5 & \\
\hline Continuous replacement therapy & 24.2 & 17.6 & 21.6 & 9.9 & \\
\hline Both (intermittent + continuous) & 11.3 & 8.1 & 3.1 & 0.0 & \\
\hline Food intake (\%) & & & & & $<0.001$ \\
\hline Optimal nutrition & 11.3 & 26.0 & 26.4 & 51.2 & \\
\hline Mild malnutrition & 19.4 & 18.3 & 17.2 & 20.2 & \\
\hline Moderate malnutrition & 22.6 & 24.4 & 39.1 & 22.6 & \\
\hline Severe malnutrition & 46.8 & 31.3 & 17.2 & 6.0 & \\
\hline
\end{tabular}


Table 3. ORs and 95\% CIs of in-hospital mortality for different Karnofsky scores

\begin{tabular}{|c|c|c|c|c|c|}
\hline \multirow[t]{2}{*}{ 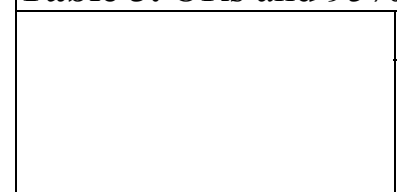 } & \multicolumn{5}{|c|}{ Karnofsky scores } \\
\hline & $\begin{array}{c}\geq 80 \\
n=7(7.7 \%)\end{array}$ & $\begin{array}{c}70 \\
n=21(21.6 \%)\end{array}$ & $\begin{array}{c}60 \\
n=54(39.7 \%)\end{array}$ & $\begin{array}{c}\leq 50 \\
n=30(48.4 \%)\end{array}$ & $\begin{array}{l}P \text { for } \\
\text { trend }\end{array}$ \\
\hline Crude OR (95\% CI) & 1.00 (Ref.) & $3.32(1.34-8.25)$ & $7.81(3.36-18.17)$ & $11.12(4.44-27.84)$ & $<0.001$ \\
\hline $\begin{array}{l}\text { Age- and sex-adjusted } \\
\text { OR }(95 \% \mathrm{CI})\end{array}$ & 1.00 (Ref.) & $3.62(1.44-9.08)$ & $8.96(3.80-21.14)$ & $12.11(4.79-30.65)$ & $<0.001$ \\
\hline $\begin{array}{l}\text { Multivariate-adjusted } \\
\text { OR* }(95 \% \mathrm{CI})\end{array}$ & 1.00 (Ref.) & $2.83(1.04-7.68)$ & $6.78(2.61-17.58)$ & $8.87(3.03-25.99)$ & $<0.001$ \\
\hline
\end{tabular}

Table 4 ORs and 95\% CIs of in-hospital mortality for every 10-point Karnofsky score

\begin{tabular}{|l|c|}
\hline Crude OR $(95 \% \mathrm{CI})$ & $1.83(1.50-2.24)$ \\
\hline Age- and sex-adjusted OR $(95 \% \mathrm{CI})$ & $1.86(1.52-2.28)$ \\
\hline Multivariate-adjusted OR* $(95 \% \mathrm{CI})$ & $1.75(1.37-2.23)$ \\
\hline
\end{tabular}

*Adjusted for age, sex, relative increment of serum creatinine, Liano scoring, RIFLE classes, food intake, basal haemoglobin, oncology patients and treatment of acute renal failure. CI, confidence interval; $\mathrm{OR}$, odds ratio. 


\section{Total patients}

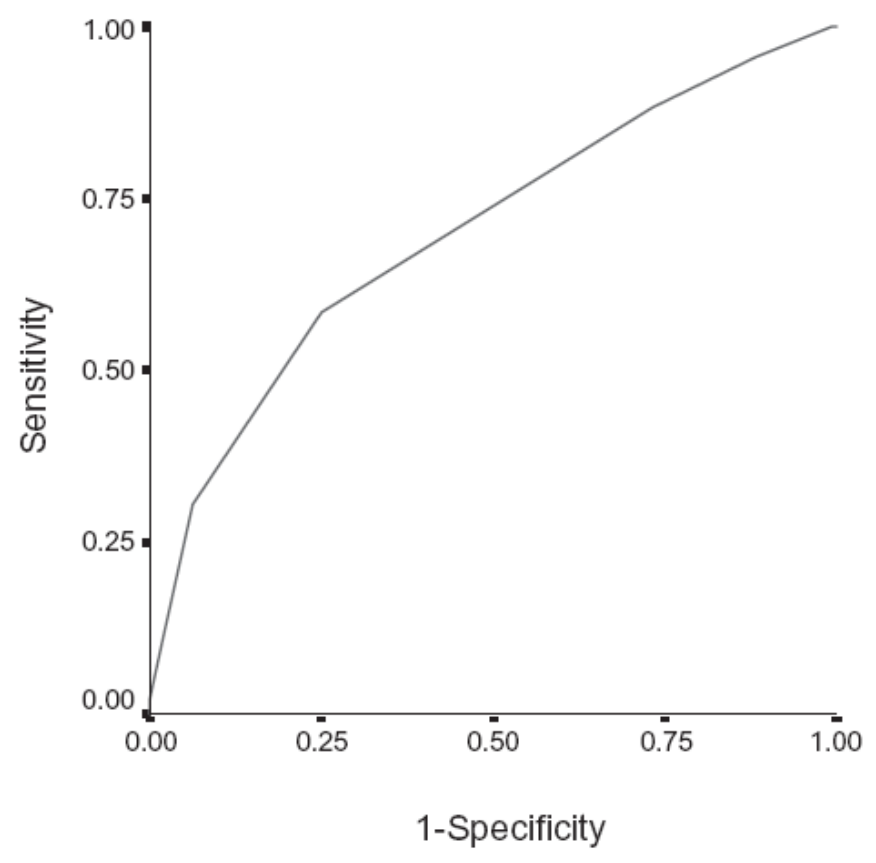

Figure 1. Receiver operating characteristic curve analysis. Area: 0.701.

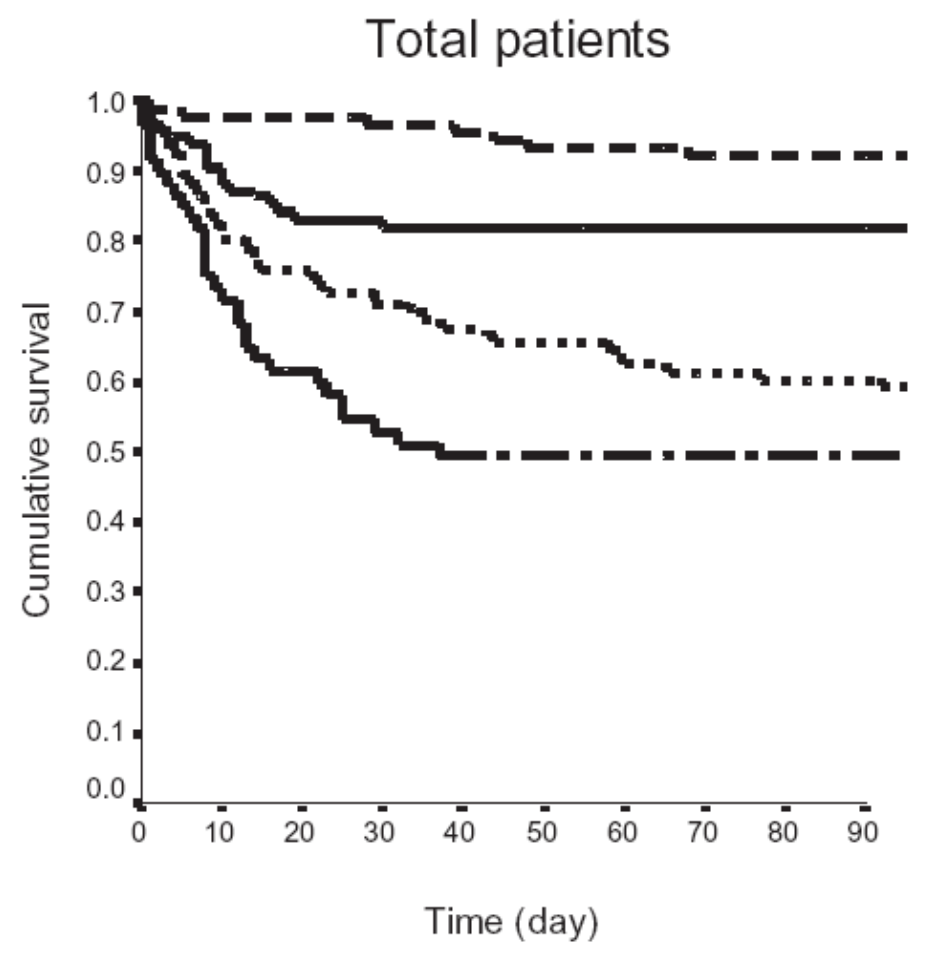

Figure 2. Cumulative survival (Log-rank test $<0.001$ ). Karnofsky groups: $--\geq 80$; $70 ; \cdots 60 ;-\cdot \leq 50$. 